\title{
Quasi-optimal synthesis of an adaptive filter in the problem of estimating the state of dynamic systems
}

\author{
Andrey Kostoglotov ${ }^{1}$, Anton Penkov ${ }^{1}$, and Sergey Lazarenko ${ }^{2, *}$ \\ ${ }^{1}$ Rostov State Transport University, 344038, Rostovskogo Strelkovogo Polka Narodnogo \\ Opolcheniya Sq., 2, Rostov-on-Don, Russia \\ ${ }^{2}$ Don State Technical University, 344003, Gagarin sq., 1, Rostov-on-Don, Russia
}

\begin{abstract}
The problem of synthesis of filters to estimate the state of dynamical systems is considered based on the condition for the maximum of the generalized power function and stationarity of the generalized Lagrangian and Hamiltonian of the estimated system model. The paper demonstrates that the use of invariants in combination with the decomposition principle makes it possible to simplify the equations of controlled motion and reduce them to a system of independent equations in terms of the number of degrees of freedom. This approach reduces the number of unknown parameters of the motion model, which greatly simplifies the adaptation process when developing filters for quasi-optimal estimation of the state parameters of dynamic systems. Comparative analysis of the results of the mathematical simulation shows that the application of the proposed method increases the efficiency of filters of the Kalman structure.
\end{abstract}

\section{Introduction}

Modern control systems set high requirements for the accuracy of the input data. Noises, systematic errors and other factors distorting the reliability of the measured data can cause failures in the operation of a single module and the entire system as a whole.

An effective solution to the problem to ensure the specified accuracy is the use of the Kalman filtering procedure [1]. Its disadvantages are due to the relatively high computational complexity, difficult determining the parameters of the mathematical model of the stochastic system, weak dependence of the feedback coefficients on observations in the steady state, etc [2]. Traditional methods of creating filters under conditions of unknown regular influences do not always allow to obtain highly accurate estimates [3].

It is possible to reduce the error and increase the stability of the estimation procedure by adapting it to significant model perturbations. Such adaptation can be realized using the methodology of the combined maximum principle [4], which leads to a model of a

*Corresponding author: lazarenkosv@icloud.com 
dynamical system that satisfies the Hamilton-Ostrogradskii principle [5]. The structure of the model is determined based on the condition of the maximum of the generalized power function up to a nonlinear synthesizing function that determines the rate of dissipation and, accordingly, the degree of structural adaptation [6]. However, the nonlinearity of the proposed model limits the possibilities of its application.

This requires the development of a new method to solve the problem to synthesize filters for estimating the state of dynamical systems based on the condition for the maximum of the generalized power function and the stationarity of the generalized Lagrangian and Hamiltonian of the model of the estimated system.

The use of invariants in combination with the decomposition principle without invoking a linear approximation makes it possible to simplify the equations of controlled motion and reduce them to a system of independent equations in terms of the number of degrees of freedom. This approach reduces the number of unknown parameters of the motion model, which greatly simplifies the adaptation process when developing filters for quasi-optimal estimation of the state parameters of dynamic systems.

The scientific task is to develop a method to synthesize a filter for quasi-optimal estimation of the state of dynamical systems with an adaptive model based on the decomposition principle with the construction of kinematic constraints based on the condition of constancy of motion invariants.

The purpose of the work is to develop a discrete filter to estimate the state and a procedure for the filter adaptation.

\section{Formulation of the problem}

The basis for construction the state estimation filter is a mathematical model of the dynamics of the process under study. In accordance with the analytical mechanics laws, the mathematical model of the motion of a dynamic system follows from the HamiltonOstrogradskii principle, according to which [7]

$$
\delta^{\prime} S=\int_{t_{0}}^{t_{1}}\left(\delta T+\delta^{\prime} A\right) d t=0
$$

for the action integral

$$
S=\int_{t_{0}}^{t_{1}}(T+A) d t
$$

where $t_{0}, t_{1}$ is the start and end time of observations.

Kinetic energy for a dynamical system with stationary constraints [7]

$$
T=\frac{1}{2} \sum_{s=1}^{n} \sum_{k=1}^{n} a_{s k} \dot{q}_{s} \dot{q}_{k}, \quad s=\overline{1, n}, \quad k=\overline{1, n},
$$

where $q_{s}, \dot{q}_{s}$ are generalized coordinates and velocities,

$a_{s k}$ are the coefficients of inertia, namely the elements of a quadratic form matrix, 
$n$ is the number of degrees of freedom.

The work of the generalized forces on the observed trajectory is determined by the following expression:

$$
A=\int_{q_{s}\left(t_{0}\right)}^{q_{s}\left(t_{1}\right)} \sum_{s=1}^{n}\left(U_{s}-\frac{\partial \Phi}{\partial \dot{q}_{s}}-\frac{\partial \Pi}{\partial q_{s}}\right) d q_{s}
$$

where $U_{s}$ are the generalized control forces,

$\frac{\partial \Phi}{\partial \dot{q}_{s}}$ are the dissipative generalized forces,

$\frac{\partial \Pi}{\partial q_{s}}$ are the potential generalized forces.

Then the Lagrange formalism allows to obtain the equations of motion in the following form $[7,8]$

$$
\begin{gathered}
\sum_{k=1}^{n} a_{k s} \ddot{q}_{k}=-\sum_{k=1}^{n} \sum_{m=1}^{n}[k, m ; s] \dot{q}_{k} \dot{q}_{m}+\left(U_{s}-\frac{\partial \Phi}{\partial \dot{q}_{s}}-\frac{\partial \Pi}{\partial q_{s}}\right)=Q_{s}(q, \dot{q}, U), \quad s=\overline{1, n}, \\
q\left(t_{0}\right)=q_{0}, \dot{q}\left(t_{0}\right)=\dot{q}_{0},
\end{gathered}
$$

where $[k, m ; s]=\frac{1}{2}\left(\frac{\partial a_{k s}}{\partial q_{m}}+\frac{\partial a_{m s}}{\partial q_{k}}-\frac{\partial a_{k m}}{\partial q_{s}}\right)$ are the Christoffel symbols of the first kind for the matrix of the quadratic form $T$.

The use of model (5) for constructing a filter for the state estimation, taking into account the completeness and detailing of the description of the process of evolution of the parameters of the object under study, is rather complicated, and such a model is not widely used in the practice of statistical synthesis.

Dynamical systems (5) are essentially nonlinear systems of a high order, which are characterized by the presence of significant dynamic interaction between elements (degrees of freedom). The intensity of mutual influence is characterized by the coefficients $a_{s k}$ in the kinetic energy model $T$. The presence of mutual influence and various disturbing factors makes difficult to solve the problem to estimate the parameters of systems described by equations (5).

One of the possible approaches to solve problems to construct models of controlled systems (without using a linear approximation) is the decomposition principle. The essence of this principle [9] is to completely eliminate the dynamic mutual influence between the elements using admissible control, to bring the system to a motion in the decomposition mode and to choose this control (eliminating mutual influence) so that the system (5) moves in accordance with the control goal, providing the given value of the functional characterizing the quality of the state assessment process. Thus, due to feedbacks, the closed system begins to move in a mode close to sliding and becomes a follower of the trajectories determined by an observer (in the sense of the estimation criterion). This means 
that the system under consideration (i.e., a nonlinear multiply connected high-order dynamical system) begins to move through a finite time interval due to the simplest system [9] This fact can be used to construct a model of the system under study.

Let us consider the mathematical formulation of the problem. The formal assignment of a part of the energy to the work of the generalized forces $Q_{s}(q, \dot{q}, U)$ during motion in the decomposition mode allows to introduce the simplest positively defined quadratic form of velocities, which is interpreted as the kinetic energy of the system (5) [10]

$$
T=\frac{1}{2} \sum_{s=1}^{n} a_{s s} \dot{q}_{s}^{2}, s=\overline{1, n} .
$$

This form is used later to build a model of the estimated process dynamics.

We assume that the observation equation has the form

$$
y=q+v,
$$

where $v$ is the vector of random actions on the observation channel with a known intensity. We choose the coordinates in which observations are made as generalized coordinates.

In the observation space we choose a target functional [5] that takes a certain specified value, which determines the accuracy of measuring the phase coordinates of the system under study

$$
J=\frac{1}{2} \int_{t_{0}}^{t_{1}} \sum_{s=1}^{n} N_{s s}^{-1}\left[y_{s}-\hat{q}_{s}\right]^{2} d t=\int_{t_{0}}^{t_{1}} F(\hat{q}) d t=D,
$$

where $N_{s s}$ are the elements of the diagonal weight matrix $\mathbf{N}$, characterizing the intensity of interference in the observation channel, the sign ${ }^{\wedge}$ means the estimate operation, $D$ is the weighted variance.

Let us formulate the problem to construct a mathematical model of the process of an observed (7) and controlled by criterion (8) dynamic system with the kinetic energy (6), taking into account that the motion of the dynamic system in the decomposition mode follows from the Hamilton-Ostrogradskii principle (1). The resulting model has an explicit relation to the gradient of the functional $(8)[7,8]$, belongs to the class of adaptive models and can be used to construct a filter for the state estimation of the Kalman structure.

\section{Building an adaptive model}

Let for the observed system (7) with kinetic energy (6) in the decomposition mode due to feedbacks the model moves in accordance with the goal which is determined by the value of the functional (8). We require that the generalized kinetic potential

$$
T-\lambda^{-1} F=0,
$$

where $\lambda(D)$ is the Lagrange multiplier acting as the model parameter. This allows to establish a kinematic constraint 


$$
\frac{1}{2} \sum_{s=1}^{n} a_{s s} \dot{q}_{s}^{2}=\lambda^{-1} \frac{1}{2} \sum_{s=1}^{n} N_{s s}^{-1}\left(y_{s}-q_{s}\right)^{2},
$$

and by the assumption that motion in the decomposition mode completely eliminates the dynamic mutual influence between the elements, we have the following relation

$$
\left|\dot{q}_{s}\right|=\sqrt{a_{s s}^{-1} \lambda^{-1} N_{s s}^{-1}}\left|y_{s}-q_{s}\right|, s=\overline{1, n} .
$$

The general form of the equation for the dynamic quasi-deterministic estimation by criterion (8) is determined as follows [6]

$$
\frac{d}{d t}\left(\frac{\partial T}{\partial \dot{q}_{s}}\right)-\frac{\partial T}{\partial q_{s}}=\lambda^{-1}\left[-\lambda \frac{\left|\dot{q}_{s}\right|}{N_{s s}^{-1}\left|y_{s}-q_{s}\right|} \dot{q}_{s}-N_{s s}^{-1}\left(y_{s}-q_{s}\right)\right], s=\overline{1, n} .
$$

Taking into account the accepted model of kinetic energy (6) and the kinematic constraint (11), we obtain the estimation equations in the form

$$
\ddot{\hat{q}}_{s}(t)=-\sqrt{\lambda_{s}^{-1}} \dot{\hat{q}}_{s}(t)-\lambda_{s}^{-1}\left(y_{s}(t)-\hat{q}_{s}(t)\right), s=\overline{1, n} .
$$

where $\lambda_{s}=a_{s s} \lambda N_{s s}$ is the adaptation coefficient.

This is an equation of the dynamical system controlled by criterion (8) with the kinetic energy (6) when moving in the decomposition mode.

Now we consider the problem to construct the Kalman filter for the equations of the model (12) and the observation (7).

\section{Discrete filter synthesis}

Equation (12) can be used to adapt the model of motion with respect to the parameter $\lambda$ for a given value of functional (8) when constructing the adaptive filter for estimation of the parameters of dynamic systems [6, 11].

Equation (12), taking into account the simplest scheme of the Euler finite-difference approximation, can be represented in the vector-matrix discrete form:

$$
\mathbf{X}(i+1)=\mathbf{F X}(i)+\mathbf{G Y}(i),
$$

where $\mathbf{X}(i)=\left[\begin{array}{l}x_{1}(i) \\ x_{2}(i)\end{array}\right]$ is the extended state vector, $x_{1}(i)=q(i), x_{2}(i)=\dot{q}(i)$,

$\mathbf{F}=\left[\begin{array}{cc}1 & \Delta t \\ \lambda^{-1} N^{-1} \Delta t & 1-\sqrt{\lambda^{-1} N^{-1}} \Delta t\end{array}\right]$ is the state transition matrix,

$\mathbf{G}=\left[\begin{array}{cc}0 & 0 \\ 0 & -\lambda^{-1} N^{-1} \Delta t\end{array}\right]$ is the excitation matrix, 
$\mathbf{Y}(i)=\left[\begin{array}{c}0 \\ y(i)\end{array}\right]$ is the observation vector,

$\Delta t$ is the sampling interval,

$\lambda$ is the adaptation coefficient,

$N$ is the spectral density of the observation noise.

Suppose that, as a result of adaptation, the value of the parameter $\lambda$ is found as a result of numerical simulation of the model (13). In this case we obtain some approximation (estimate) of the true value of the parameter $\hat{q} \approx q$. Then from equation (12) we obtain a stochastic differential equation, which serves as the basis for constructing a model of the dynamics of continuous stochastic systems and can be described by the nonstrict Langevin equation [5]

$$
\ddot{\hat{q}}(t)=-\sqrt{\lambda^{-1} N^{-1}} \dot{\hat{q}}(t)-\lambda^{-1} N^{-1} w(t),
$$

where $\hat{q}(t)$ is the estimation of the system state,

$w(t)$ is the input noise [1].

Since in the decomposition mode the dynamic mutual influence of the degrees of freedom is eliminated (compensated), the system begins to move due to a system of unlinked non-interacting subsystems. This allows to consider a one-dimensional case. The observation for the one-dimensional case is described by equation (7).

In accordance with [4] the solution of the filter problem requires the use of a formal procedure for expanding the state space, which allows to write (14) in the form of a firstorder vector differential equation:

$$
\dot{\mathbf{X}}(t)=\boldsymbol{\Phi} \mathbf{X}(t)+\Gamma \mathbf{W}(t), \mathbf{X}(0)=\mathbf{X}_{0}
$$

where $\boldsymbol{\Phi}=\left[\begin{array}{cc}0 & 1 \\ 0 & -\sqrt{\lambda^{-1} N^{-1}}\end{array}\right], \quad \boldsymbol{\Gamma}=\left[\begin{array}{cc}0 & 0 \\ 0 & -\lambda^{-1} N^{-1}\end{array}\right]$,

$\mathbf{X}_{0}$ is the vector of initial conditions,

$\mathbf{W}(t)$ is the vector of random actions with covariance matrix $\mathbf{Q}$.

Discrete model for (15) is:

$$
\begin{gathered}
\mathbf{X}(i+1)=\mathbf{F X}(i)+\mathbf{G W}(i) \\
\mathbf{F}=\left[\begin{array}{cc}
1 & \Delta t \\
0 & 1-\sqrt{\lambda^{-1} N^{-1}} \Delta t
\end{array}\right], \mathbf{G}=\left[\begin{array}{cc}
0 & 0 \\
0 & -\lambda^{-1} N^{-1} \Delta t
\end{array}\right], \mathbf{Q}=\left[\begin{array}{cc}
0 & 0 \\
0 & \lambda^{-2} N^{-2} \Delta t^{2}
\end{array}\right]
\end{gathered}
$$

Observation equation (7) in discrete form is:

$$
\mathbf{Y}(i)=\mathbf{H X}(i)+\mathbf{V}(i)
$$

where $\mathbf{V}(i)$ is the vector of measurement error with the covariance matrix $\mathbf{R}(i)$, 
$\mathbf{H}=\left[\begin{array}{ll}1 & 0 \\ 0 & 0\end{array}\right]$ is the projection of the state space onto the observation space.

Equation (16) has a form that allows to synthesize the Kalman filter [12].

For the classical Kalman filter, the corresponding matrices [12]:

$$
\mathbf{F}=\left[\begin{array}{cc}
1 & \Delta t \\
0 & 1
\end{array}\right], \mathbf{G}=\left[\begin{array}{cc}
0 & 0 \\
0 & \Delta t
\end{array}\right], \mathbf{Q}=\left[\begin{array}{cc}
0 & 0 \\
0 & \Delta t^{2}
\end{array}\right]
$$

According to the generalized Bayesian estimation scheme, the Kalman filter consists of two series-connected devices: the extrapolator that predicts the state of an object one step ahead, and the filter that refines the extrapolated value based on new measurement [12].

The discrete Kalman filter algorithm has the form:

$$
\begin{gathered}
\hat{\mathbf{X}}(i \mid i-1)=\mathbf{F} \hat{\mathbf{X}}(i-1) \\
\hat{\mathbf{X}}(i)=\hat{\mathbf{X}}(i \mid i-1)+\mathbf{K}(i)(\mathbf{Y}(i)-\mathbf{H} \hat{\mathbf{X}}(i \mid i-1)), \\
\mathbf{K}(i)=\mathbf{P}(i) \mathbf{H}^{T} \mathbf{R}^{-1} \\
\mathbf{P}(i)=(\mathbf{I}-\mathbf{K}(i) \mathbf{H}) \mathbf{P}(i \mid i-1), \\
\mathbf{P}(i \mid i-1)=\mathbf{F} \mathbf{P}(i-1) \mathbf{F}^{T}+\mathbf{G Q G} \mathbf{G}^{T}
\end{gathered}
$$

where $\quad \hat{\mathbf{X}}(i)$ is the estimate of the state vector, $\hat{\mathbf{X}}(i \mid i-1)$ is the extrapolated state vector estimate, $\mathbf{P}(i)$ is the state vector estimate covariance, $\mathbf{P}(i \mid i-1)$ is the extrapolated state vector error covariance, $\mathbf{K}(i)$ is the filter matrix gain, $\mathbf{I}$ is the unity matrix.

\section{The procedure for adapting the model and evaluating the efficiency of the developed filter}

Let us consider a dynamic system associated with the estimation of the angular position of an unmanned aerial vehicle (UAV) by the roll angle [13]. In the case when the UAV is in a stationary mode, the traditional Kalman filter makes it possible to achieve a satisfactory accuracy in estimating the angular position. However, in the case of maneuvers or external forces (for example, atmospheric turbulence), the accuracy of the estimate decreases [14, $15]$.

An experiment was carried out to determine the filtration efficiency by the algorithm (19) with the adaptive model (16) and with the traditional model (18), as well as to compare the results obtained with the corresponding estimate by the adaptive extrapolator (13).

Let the measuring channel form the data for determining the angle by an accelerometer, which design feature is a high level of noise. Let us consider test modes determined by stepwise and oscillatory disturbing actions onto the dynamic system:

a) Stepwise action

Initial data for numerical simulation: 


$$
\begin{gathered}
\mathbf{X}(1)=\left[\begin{array}{l}
0 \\
0
\end{array}\right], \mathbf{P}(1 \mid 1)=\left[\begin{array}{cc}
1 & 0.5 \\
0.5 & 1
\end{array}\right], \\
\Delta t=0.02 \mathrm{c}, \sigma_{v}=9^{\circ}(60 \% \text { of the amplitude of the action }),
\end{gathered}
$$

where $\sigma_{v}$ is the standard deviation of the observation noise.

As a result of the model adaptation by functional (8), we obtain the value of the adaptation parameter of $\lambda=0.005$.

The results of the extrapolator and filters operation are shown in Figure 1, where the abscissa indicates the time in seconds, the ordinate indicates the roll angle, the numbers indicate:

1 - adaptive extrapolator (13);

2 - evaluation of the proposed algorithm (16);

3 - evaluation of the traditional Kalman filter (18).

4 - initial perturbation.

b) Oscillatory action

Initial data for numerical simulation:

$$
\begin{gathered}
\mathbf{X}(1)=\left[\begin{array}{l}
0 \\
0
\end{array}\right], \mathbf{P}(1 \mid 1)=\left[\begin{array}{cc}
1 & 0.5 \\
0.5 & 1
\end{array}\right], \\
\Delta t=0.02 \mathrm{c}, \sigma_{v}=9^{\circ}(60 \% \text { of the amplitude of the action }) .
\end{gathered}
$$

As a result of the model adaptation by functional (8), we obtain the value of the adaptation parameter of $\lambda=0.0025$. 1.

The simulation results are shown in Figure 2, where the notations correspond to Figure

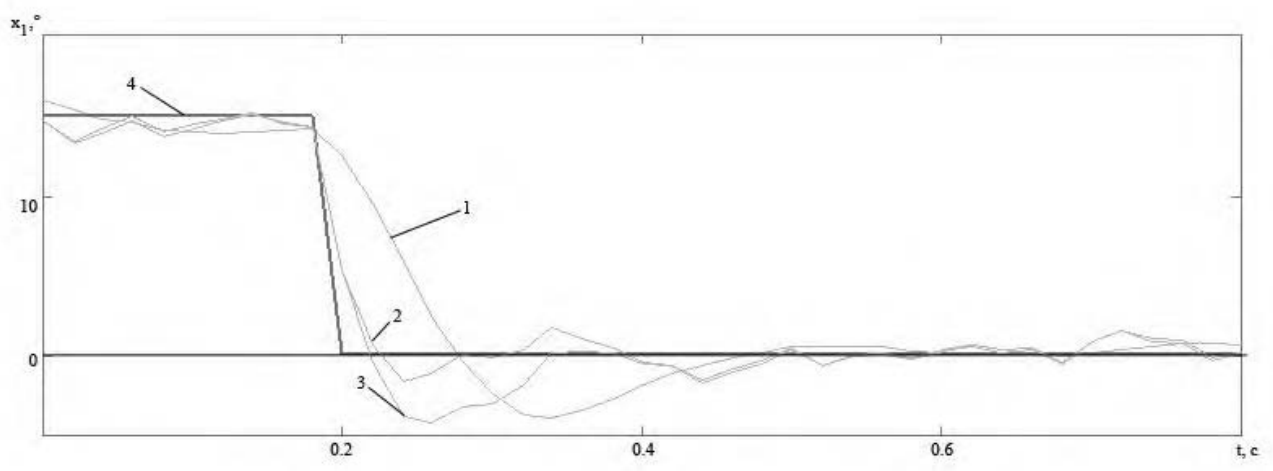

Fig. 1.Transient process in the case of stepwise action. 


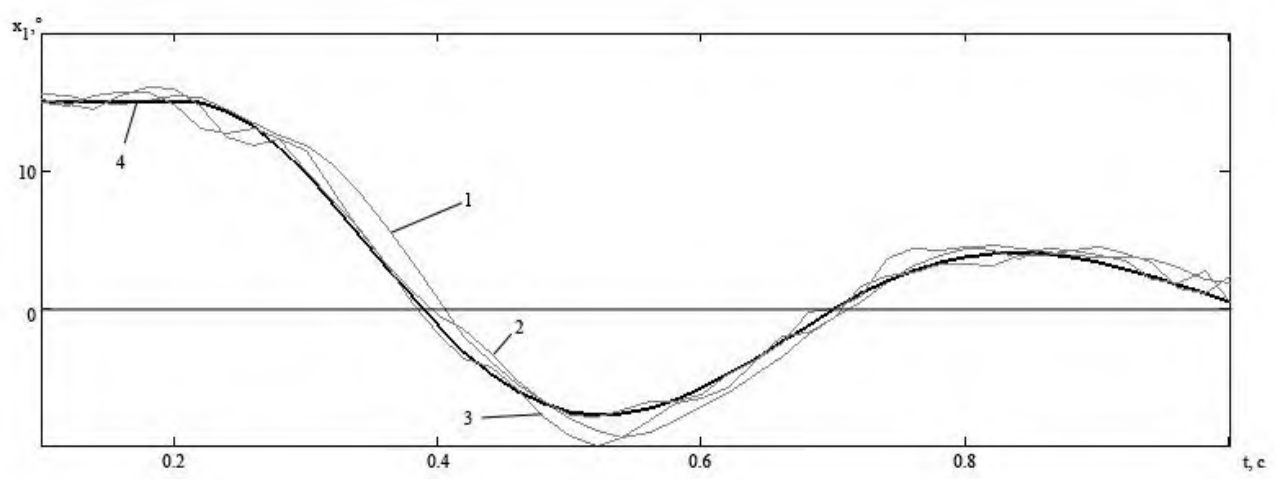

Fig. 2.Transient process in the case of oscillatory action.

The averaged RMS deviations of the estimates of the UAV roll angle under various action modes are given in Tables 1 and 2 .

Table 1.RMS deviation of the roll angle estimation under stepwise action

\begin{tabular}{|c|c|c|c|}
\hline & $\begin{array}{c}\text { Classic model } \\
(\mathbf{1 8 )}\end{array}$ & $\begin{array}{c}\text { Adaptive model } \\
(\mathbf{1 6})\end{array}$ & Extrapolator (13) \\
\hline $\begin{array}{c}\text { Over the entire } \\
\text { observation interval }\end{array}$ & $3.21^{\circ}$ & $2.6^{\circ}$ & $3.01^{\circ}$ \\
\hline On the transient interval & $3.75^{\circ}$ & $3.49^{\circ}$ & $3.85^{\circ}$ \\
\hline
\end{tabular}

Table 2.RMS deviation of roll angle estimation under oscillatory action

\begin{tabular}{|c|c|c|c|}
\hline & $\begin{array}{c}\text { Classic model } \\
(\mathbf{1 8 )}\end{array}$ & $\begin{array}{c}\text { Adaptive } \\
\text { model (16) }\end{array}$ & $\begin{array}{c}\text { Extrapolator } \\
\text { (13) }\end{array}$ \\
\hline $\begin{array}{c}\text { Over the entire } \\
\text { observation interval }\end{array}$ & $3.99^{\circ}$ & $3.33^{\circ}$ & $4.18^{\circ}$ \\
\hline On the transient interval & $4.06^{\circ}$ & $3.31^{\circ}$ & $3.89^{\circ}$ \\
\hline
\end{tabular}

The study of each of the described algorithms is carried out for various RMS deviations of observation noise $(10 \%, 20 \%, 40 \%$, and $60 \%$ of the amplitude of the action), with 25 realizations for each value. The dependences of the RMS deviation of the estimate (along the ordinate) on the RMS deviation of the observation noise (along the abscissa) are shown in Figures 3 and 4, where the numbers indicate:

1 - RMS deviation of the adaptive extrapolator (13),

2 - RMS deviation of the adaptive filter (16),

3 - RMS deviation of the traditional Kalman filter (18). 


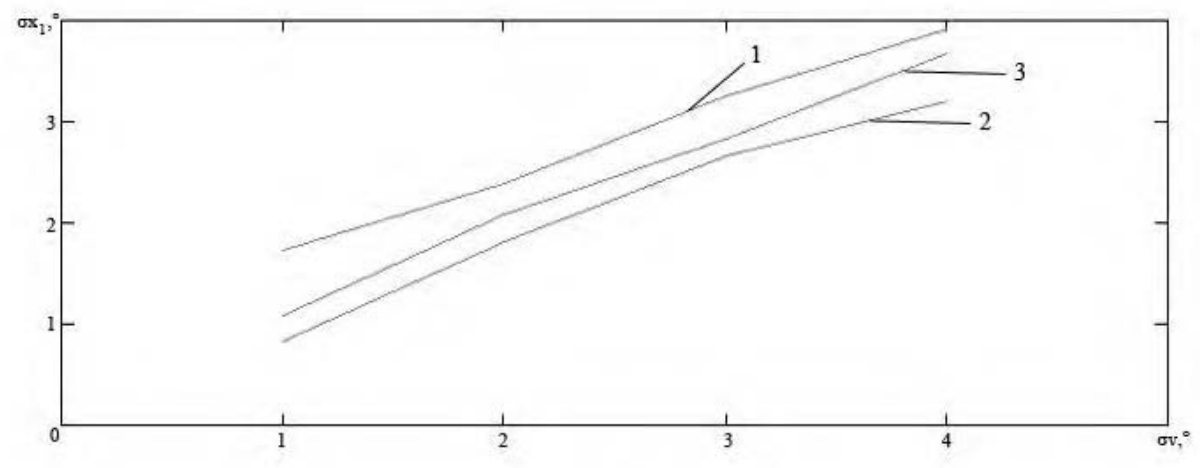

Fig. 3.RMS deviation of the roll angle estimation under stepwise action

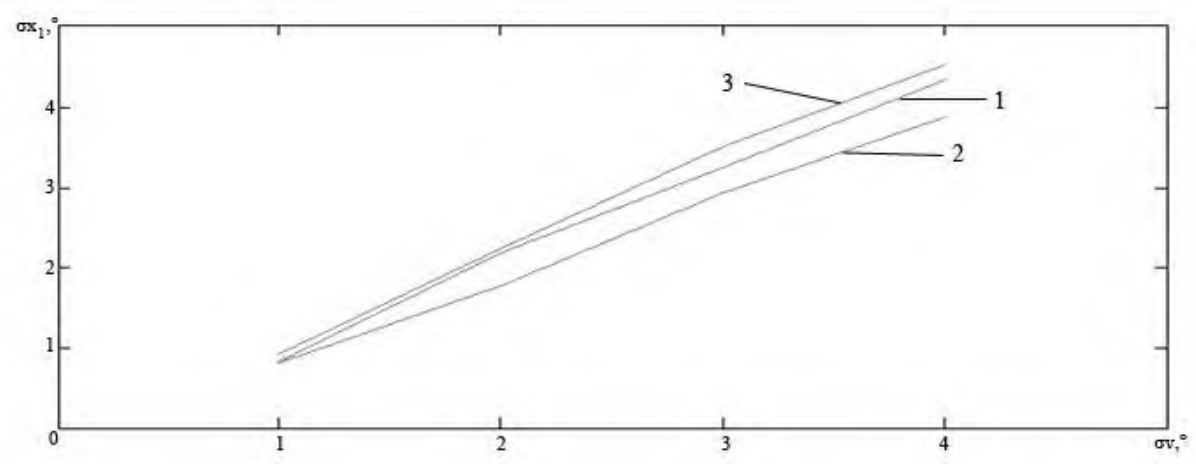

Fig. 4. RMS deviation of roll angle assessment under oscillatory action.

\section{Conclusions}

The results obtained allow us to conclude that the estimation error is reduced when using the adaptive model as part of the filter. An increase in accuracy is observed in a wide range of observation noise, which indicates effectiveness of the developed method and practicability to use the quasi-optimal adaptive algorithms based on the condition for the maximum function of the generalized power in filtering systems. $00385 \mathrm{~A}$.

The reported study was funded by RFBR, project number 19-38-90273, 18-08-01494 A, 18-01-

\section{References}

1. A. Zulu, S. John, A review of control algorithm for autonomous quadrotors, inOpen Journal of Applied Sciences, 4, 547-556 (2014) doi: 10.4236/ojapps.2014.414053.

2. Z. Li, S. Chen, H. Leung, E. Bosse, Joint Data Association, Registration, and Fusion using EM-KF, in IEEE Transactions on Aerospace and Electronic Systems, 2, 496-507, (2010) doi: 10.1109/TAES.2010.5461637. 
3. X. Yuan, F. Lian, C. Han, Models and Algorithms for Tracking Target with Coordinated Turn Motion, in Mathematical Problems in Engineering, Article ID 649276, 10 (2014) doi: 10.1155/2014/649276.

4. A. Kostoglotov, S. Lazarenko, A. Penkov, I. Kirellov, O. Manaenkova, Synthesis of adaptive algorithms for estimating the parameters of angular position based on the combined maximum principle, in Advances in Intelligent Systems and Computing, 873, 107-115 (2019) doi: 0.1007/978-3-030-01818-4_11.

5. A. Kostoglotov, I. Pugachev, A. Penkov, S. Lazarenko, Synthesis of Multi-model Algorithms for Intelligent Estimation of Motion Parameters Under Conditions of Uncertainty Using Condition of Generalized Power Function Maximum and Fuzzy Logic, in Advances in Intelligent Systems and Computing, 1156, 541-547 (2020) doi: 10.1007/978-3-030-50097-9_55.

6. A. Kostoglotov, S. Lazarenko, A. Kuznetsov, I. Deryabkin, V. Losev, Structural synthesis of discrete adaptive tracking systems based on the combined maximum principle, in Vestnik of Don State Technical University, 17, 105-112 (2017) doi: 10.23947/1992-5980-2017-17-1-105-113.

7. W. Nolting, Theoretical Physics 2. Analitical mechanics, 358 (2016) doi: 10.1007/9783-319-40129-4

8. T. Luukkonen, Modelling and control of quadcopter, in Independent research project in applied mathematics, Article ID 2. 4108, 24 (2011)

9. A. Kostoglotov, I. Derabkin, S. Lazarenko, Identification of design parameters using the combined maximum principle and the decomposition principle, in 2017 International Conference on Industrial Engineering, Applications and Manufacturing, 1-7, 10.1109/ICIEAM.2017.8076165

10. R. Jazar, Theory of applied robotics. Kinematics, Dynamics, and Control (2nd edition), in Springer, 693 (2010)

11. S. Kangwague, J. W. Choi, Design of a tracking filter suitable for 2D motion dynamics of an off-road target, in 17th International Conference on Control, Automation and Systems (ICCAS), 379-382 (2017) doi: 10.23919/ICCAS.2017.8204469.

12. A. Konovalov, Basics of processing trajectory information, 164 (2013)

13. D. Kingston, R. Beard, Real-Time Attitude and Position Estimation for Small UAVs Using Low-Cost Sensors, 1-9 (2014) doi: 10.2514/6.2004-6488.

14. A. Chovancová, T. Fico, L. Chovanec, P. Hubinský, Mathematical Modelling and Parameter Identification of Quadrotor (a survey), in Procedia Engineering, 96, 172181 (2014) doi: 10.1016/j.proeng.2014.12.139

15. A. Kostat, G. Nikolakopoulos, A. Tzes, Switching model predictive attitude control for a quadrotor helicopter subject to atmosperic disturbances, in Control engineering, 19, 1195-1207 (2011) 